\title{
Effect of hypoxia and glutamine or glucose deprivation on the expression of retinoblastoma and retinoblastoma-related genes in ERN1 knockdown glioma U87 cell line
}

\author{
Dmytro O. Minchenko ${ }^{1,2,3^{*}}$, Leonid L. Karbovskyi ${ }^{1}$, Serhii V. Danilovskyi ${ }^{1}$, Michel Moenner ${ }^{3}$, \\ Oleksandr H. Minchenko ${ }^{1,3}$ \\ ${ }^{1}$ Department of Molecular Biology, Palladin Institute of Biochemistry National Academy of Sciences of Ukraine, Kyiv, Ukraine \\ ${ }^{2}$ National O.O. Bohomoletz Medical University, Kyiv, Ukraine \\ ${ }^{3}$ INSERM U920 Molecular Mechanisms of Angiogenesis Laboratory, University Bordeaux 1, Talence, France \\ Email: ${ }^{*}$ ominchenko@yahoo.com, xanrok@yahoo.com
}

Received 2 August 2011; revised 12 September 2011; accepted 26 September 2011

\begin{abstract}
The expression of retinoblastoma and several retinoblastoma-related genes was studied in glioma cell line U87 and its subline with knockdown of ERN1 (endoplasmic reticulum-nuclei-1), the main endoplasmic reticulum stress sensing and signaling enzyme. It was shown that a blockade of the ERN1 enzyme function increases the expression levels of retinoblastoma, retinoblastoma-like 1 and most retinoblastoma related genes: EID1, JARID1B, E2F1, E2F3, RBAP48 and CTIP, does not change RNF40 and RBAP46 and decreases KDM5A. We have also demonstrated that hypoxia reduces the expression levels of retinoblastoma, EID1, and E2F1 in ERN1-deficient glioma cells only. At the same time, the expression levels of retinoblastoma-like 1, E2F3, RBAP46, RBAP48 and CTIP decrease, while JARID1B and RBBP2 increase in both types of cells in hypoxic conditions, but the expression is much stronger in cells with suppressed function of ERN1. The expression level of JARID1B and KDM5A mRNA is also enhanced in glutamine deprivation condition in both tested cell types, moreover, this effect is amplified by the blockade of the ERN1 enzyme function. The expression levels of retinoblastoma, EID1, RBAP48, and E2F3 are decreased in glutamine deprivation condition only in ERN1-deficient glioma cells, but RBL1, CTIP, RBAP46, and E2F1-in both tested cell types with more significant effect in ERN1deficient cells. Glucose deprivation condition leads to a decrease of expression levels of retinoblastoma, RBL1, E2F3, RBAP46, and RBAP48 in both used cell types and of EID1 and E2F1 only in glioma cells with suppressed function of signaling enzyme ERN1. Thus,

* corresponding author.
\end{abstract}

expression levels of retinoblastoma and most retinoblastoma-related genes are increased under a blockade of ERN1 enzyme function and significantly changed in hypoxia, glucose or glutamine deprivation conditions both in control U87 cells and ERN1-deficient cells, but inhibition of the unfolded protein response sensor ERN1 predominantly enhances these effects. Moreover, it is possible that the induction of the expression of retinoblastoma and most retinoblastomarelated genes after knockdown of ERN1 plays an important role in suppression of glioma proliferation.

Keywords: mRNA Expression; Retinoblastoma, RBL1, RBAP48; RBAP46; CTIP; KDM5A; JARID1B; E2F1; E2F3; Glioma Cells; ERN1; Hypoxia; Glucose

Deprivation; Glutamine Deprivation

\section{INTRODUCTION}

The endoplasmic reticulum is a key organelle in the cellular response to ischemia, hypoxia, and some chemicals which activate a complex set of signaling pathways named the unfolded protein response. This adaptive response is activated upon the accumulation of misfolded proteins in the endoplasmic reticulum and is mediated by three endoplasmic reticulum-resident sensors named PERK (PRK-like ER kinase), IRE1/ERN1 (Inositol Requiring Enzyme-1/Endoplasmic Reticulum-Nuclei-1) and ATF6 (Activating Transcription Factor 6), however, endoplasmic reticulum-nuclei-1 is the dominant sensor [1-5]. Activation of the unfolded protein response tends to limit the de novo entry of proteins in to the endoplasmic reticulum and facilitate both the endoplasmic reticulum protein folding and degradation to adapt cells 
for survival or, alternatively, to enter cell death programs through endoplasmic reticulum-associated machineries $[1,2,4]$. As such, it participates in the early cellular response to the accumulation of misfolded proteins in the lumen of the endoplasmic reticulum, occurring under both physiological and pathological conditions.

Two distinct catalytic domains of the bifunctional signaling enzyme endoplasmic reticulum - nuclei-1 were identified: a serine/threonine kinase and an endoribonuclease which contribute to ERN1 signalling. The ERN1associated kinase activity autophosphorylates and dimerizes this enzyme, leading to the activation of its endoribonuclease domain, degradation of a specific subset of mRNA, and initiation of the pre-XBP1 (X-box binding protein 1) mRNA splicing [6-8]. Mature XBP1 mRNA splice variant encodes a transcription factor that has different C-terminus amino acid sequence and stimulates the expression of hundreds of unfolded protein responsespecific genes [1,9-11].

Recently single mutations were detected in endoplasmic reticulum - nuclei-1 gene of different human cancers and encoded by this gene enzyme was proposed as a major contributor to tumor (including glioblastoma) progression among protein kinases [12]. Moreover, the growing tumor requires the endoplasmic reticulum stress as well as hypoxia and ischemia for own neovascularization and growth and the complete blockade of ERN1 signal transduction pathway has anti-tumor effects [13-16]. The endoplasmic reticulum stress response-signalling pathway is linked to the neovascularization process, tumor growth and differentiation as well as cell death processes $[15,17,18]$. Thus, the blockade of the main unfolded protein response sensor ERN1 is important in studying the role of ERN1 signalling pathways in tumor progression, especially in malignant gliomas; it is important in developing a new understanding concerning molecular mechanisms of malignant tumors progression in relation to ischemia/hypoxia and it will help define the best targets for the design of specific inhibitors that could act as potent antitumor drugs. Gliomas are the most frequent primary brain neoplasms and represent a major challenge in cancer therapy as they are not easily accessible to current therapies.

Retinoblastoma proteins and family of retinoblastoma associated and binding proteins participate in the control of the cell cycle, proliferation, apoptosis, and differentiation $[19,20]$. The retinoblastoma-1 (RB1) gene product is a tumor suppressor protein that appears to be involved in cell cycle regulation, as it is phosphorylated in the $\mathrm{S}$ to $\mathrm{M}$ phase transition and is dephosphorylated in the G1 phase of the cell cycle. The retinoblastoma protein has been shown to interact with cyclin D1 protein [20]. The expression of cyclin D1 gene is regulated positively by retinoblastoma. This protein negatively regulates the
G1-S transition by binding to the E2F transcription factors, until the cyclin-dependent kinases phosphorylate the retinoblastoma protein, causing E2F release. For tumor growth suppression high-affinity interaction between C-terminal domain of retinoblastoma and E2F-differentiation protein (DP) heterodimer transcription complex is required. Retinoblastoma-E2F1 complex plays a critical role in ZBRK1 transcriptional repression, the loss of which may contribute to cellular sensitivity of DNA damage, ultimately leading to carcinogenesis [21].

Active E2F1/DP1 promotes apoptosis in both a p53dependent and independent manner, induces the expression of ADP ribosylation factor, which in turn blocks MDM2-mediated ubiquination of $\mathrm{p} 53$, or enhances $\mathrm{p} 53$ transcriptional activity via direct binding of E2F1 to p53 [22]. The cyclin D3 protein has been shown to interact with and be involved in the phosphorylation of tumor suppressor retinoblastoma protein. There is data that retinoblastoma controls nuclear receptor networks critical for tumor progression and that it does so via E2F transcription factor 1-mediated regulation of androgen receptor expression and output [23]. Moreover, the p16(INK4a)/ retinoblastoma pathway participates in the regulation of cellular senescence caused by damaging agents [24]. The protein encoded by retinoblastoma like 1 (RBL1) gene is similar in sequence and possibly function to the product of the RB1 gene. Both the RB1 protein and the product of RBL1 gene can inhibit the transcription of cell cycle genes containing E2F binding sites in their promoters.

The family of retinoblastoma binding or associated proteins participates in the control of cell cycle, proliferation, and differentiation, but these proteins have different functions. The $95 \mathrm{kDa}$ retinoblastoma-associated protein contains a RING finger, a motif known to be involved in protein-protein and protein-DNA interaction, and has E3 ubiquitin-protein ligase activity. The retinoblastoma binding protein-2 (KDM5A) and retinoblastoma binding protein-2H1 (JARID1B or KDM5B) are ubiquitously expressed nuclear proteins with lysine-specific demethylase activities and exert inhibitory effects on multiple genes through direct interaction with their promoters [25]. Thus, PARP-1 regulates chromatin structure and transcription through a JARID1B-dependent pathway [26]. The histone demethylase JARID1B is involved in the proliferation of cancer cells through the E2F/retinoblastoma pathway [27]. The retinoblastoma-binding proteins p48 (RBAP48) and p46 (RBAP46) are present in protein complexes involved in histone acetylation and chromatin assembly as well as is a part of co-repressor complexes, which is an integral component of transcriptional silencing, and seems to be involved in transcriptional repression of E2F-responsive genes [28]. Moreover, RBAP46 and RBAP48 interact with estrogen receptor alpha at endogenous, estrogen-responsive genes 
and alter expression of estrogen receptor alpha-activated and estrogen receptor alpha-repressed genes in MCF-7 breast cancer cells [29]. The CTBP-interacting protein (CTIP) is a retinoblastoma-binding and ubiquitously expressed nuclear protein which has DNA endonuclease activity [30]. There is data that double-strand break resection protein CTIP is a SIRT6 interaction partner and that SIRT6 promotes DNA end resection through CTIP deacetylation [31]. CTIP has been identified as a key substrate by which SIRT6 facilitates DSB processing and homologous recombination. Moreover, it is associated with BRCA1 (breast cancer 1) and is thought to modulate the functions of BRCA1 in transcriptional regulation, DNA repair, and/or cell cycle checkpoint control.

The main goal of this work is to study the role of ERN1-signaling pathways in tumor progression by investigation of the expression of tumor suppressor gene retinoblastoma and retinoblastoma-associated or binding proteins in U87 glioma cells and its ERN1-deficient subline under normal, hypoxic and ischemic (glucose or glutamine deprivation) conditions.

\section{MATERIALS AND METHODS}

\subsection{Cell Lines and Culture Conditions}

The glioma cell line U87 was obtained from ATCC (USA) and grown in high glucose (4.5 g/l) Dulbecco's modified Eagle's minimum essential medium (DMEM; Gibco, Invitrogen, USA) supplemented with glutamine (2 mM), $10 \%$ fetal bovine serum (Equitech-Bio, Inc., USA), penicillin (100 units/ml; Gibco) and streptomycin $(0,1 \mathrm{mg} / \mathrm{ml}$; Gibco) at $37^{\circ} \mathrm{C}$ in a $5 \% \mathrm{CO}_{2}$ incubator. In this work we used two sublines of this glioma cell line. One subline was obtained by selection of stable transfected clones with overexpression of vector, which was used for creation of dnERN1. This untreated subline of glioma cells (control glioma cells) was used as control 1 in the study of the effect of hypoxia and glutamine or glucose deprivations on the expression level of retinoblastoma and retinoblastoma-related genes. Second subline was obtained by selection of stable transfected clones with overexpression of ERN1 dominant/negative constructs (dnERN1) and has suppressed both protein kinase and endoribonuclease activities of this signaling enzyme [13]. The expression level of retinoblastoma and retinoblastomarelated genes in these cells was compared with cells, transfected by vector (control 1), but this subline was also used as control 2 for investigation the effect of hypoxia and glutamine or glucose deprivations on the expression level of retinoblastoma and retinoblastomarelated genes under blockade ERN1 function.

Hypoxic conditions were created in special incubator with 3\% oxygen and 5\% carbon dioxide levels; culture plates were exposed to these conditions for $16 \mathrm{hrs}$. For glucose or glutamine deprivation the growing medium in culture plates was replaced with a medium without glucose or without glutamine and thus exposed for $16 \mathrm{hrs}$.

\subsection{RNA Isolation}

Total RNA was extracted from different tumor tissues and normal tissue counterparts as described $[32,33]$. RNA pellets were washed with $75 \%$ ethanol and dissolved in nuclease-free water.

\subsection{Reverse Transcription and Quantitative PCR Analysis}

The expression levels of cyclin retinoblastoma, retinoblastoma like-1, retinoblastoma associated protein RNF40 (ring finger protein 40), retinoblastoma associated protein EID1 (EP300 interacting inhibitor of differentiation 1), retinoblastoma binding protein-2 KDM5A (lysinesspecific demethylase 5A), histone demethylase JARID1B or KDM5B, histone binding protein p48 (RBAP48), retinoblastoma binding protein p46 (RBAP46) and CTBPinteracting protein (CTIP) mRNA were measured in glioma cell line U87 and its subline with a deficiency of endoplasmic reticulum - nuclei-1 by quantitative polymerase chain reaction of complementary DNA (cDNA) using "Stratagene Mx 3000P cycler" (USA) and SYBRGreen Mix (AB gene, Great Britain). QuaniTect Reverse Transcription Kit (QIAGEN, Germany) was used for cDNA synthesis as described previously [33]. Polymerase chain reaction was performed in triplicate. For amplification of retinoblastoma (RB1) cDNA we used forward (5'-TGCATGGCTCTCAGATTCAC-3' and reverse (5'-AAGGCTGAGGTTGCTTGTGT-3') primers. The nucleotide sequences of these primers correspond to sequences 1849 - 1868 and 2073 - 2054 of human RB1 cDNA (GenBank accession number NM_000321). The amplification of retinoblastoma like-1 (RBL1) cDNA was performed using forward primer (5'-GAAGGGATGTTCGAGGACAA-3') and reverse primer (5'TGCGGCATGCAACATATAAT-3'). These oligonucleotides correspond to sequences $74-93$ and $284-265$ of human RBL1 cDNA (GenBank accession number NM 002895). The amplification of retinoblastoma associated protein RNF40 (ring finger protein 40) cDNA for real time RCR analysis was performed using two oligonucleotides primers: forward-5'-TTATTGCTGGAGCTGCCTTT-3' and reverse-5'-CTCTGGTGGGTTGAGGAGAG-3'. The nucleotide sequences of these primers correspond to sequences 1366 - 1385 and 1589 - 1570 of human RNF40 cDNA (GenBank accession number NM_014771). Two other primers were used for real time RCR analysis of the expression of retinoblastoma associ- 
ated protein EID1 (EP300 interacting inhibitor of differentiation 1) cDNA: forward-5'-GCGGGTTTCAGATGCATTAT-3' and reverse-5'-AGTTGGGTCCCTCCTCAAGT-3'. The nucleotide sequences of these primers correspond to sequences 518 - 537 and 696 - 677 of human EID1 cDNA (GenBank accession number NM_014335). For amplification of retinoblastoma binding protein-2 (KDM5A; lysines-specific demethylase 5A) cDNA we used forward (5'-CAACGGAAAGGCACT-CTCTC-3' and reverse (5'-CAAAGGCTTCTCGAGG-TTTG-3') primers. The nucleotide sequences of these primers correspond to sequences 1204 - 1223 and 1420 - 1401 of human KDM5A cDNA (GenBank accession number NM_005056). For amplification of histone demethylase 5B (JAKID1B; lysines-specific demethylase 5B) cDNA we used forward (5-GGTGAGCCAAAAACCTGGTA-3' and reverse (5'-AATCACAAACTCCCCAGCAC-3') primers. The nucleotide sequences of these primers correspond to sequences 1656 - 1675 and 1856 - 1837 of human JAKID1B cDNA (Gen-Bank accession number NM_006618). For real time RCR analysis of transcription factor E2F1 (retinoblastoma binding protein-3) cDNA expression we used next primers: forward -5'--GGGCTCTAACTGCACTTTCG-3' and reverse-5'AGGGAGTTGGGGTATCAACC-3'. The nucleotide sequences of these primers correspond to sequences 1830 1849 and 2096 - 2077 of human E2F1 cDNA (GenBank accession number NM_005225). For real time RCR analysis of transcription factor E2F3 cDNA expression we used next primers: forward-5'-GATGGGGTCAGATGGAGAGA-3' and reverse-5'-GAGACACCCTGGCATTGTTT-3'. The nucleotide sequences of these primers correspond to sequences 2931 - 2950 and 3141 - 3122 of human E2F3 cDNA (GenBank accession number NM_ 001949). For amplification of histone binding protein p48 (RBAP48; chromatin assembly factor) cDNA we used forward (5'-GATGACCCATGCTCTGGAGT-3' and reverse (5'-CGCATCAAACTGAGCATCAT-3') primers. The nucleotide sequences of these primers correspond to sequences 264 - 283 and 444 - 425 of human RBAP48 cDNA (GenBank accession number NM_ 005610). The amplification of retinoblastoma binding protein p46 (RB-AP46; histone acetyltransferase type B, subunit 2) cDNA we used forward (5'-TTCAGTGGCCCAGTCTTACC-3' and reverse (5'-CAGAACCAAAGCCACCAAAT-3') primers. The nucleotide sequences of these primers correspond to sequences $474-493$ and 686 - 667 of human RBAP46 cDNA (GenBank accession number NM_002893). Two other primers were used for real time $\overline{\mathrm{RCR}}$ analysis of $\mathrm{CTBP}$-interacting protein (CTIP; DNA endonuclease) cDNA: forward (5'- CCGAACATCCAAAAGGAAGA-3' and reverse (5'-CCTTGGCTTTTCTCTTGACG-3') primers. The nucleotide sequences of these primers correspond to sequences 1657 - 1676 and 1854 - 1835 of human CTIP cDNA (GenBank accession number NM_002894). The amplification of beta-actin cDNA was performed using forward-5'-CGTACCACTGGCATCGTGAT-3' and reverse-5'-GTGTTGGCGTACAGGTCTTT-3' primers. The expression of beta-actin mRNA was used as control of analyzed RNA quantity. The primers were received from "Sigma" (USA).

An analysis of quantitative PCR was performed using special computer program "Differential expression calculator" and statistic analysis - in Excel program. The amplified DNA fragments were separated on a $2 \%$ agarose gel and that visualized by $5 \mathrm{x}$ Sight DNA Stain (EUROMEDEA).

\section{RESULTS}

In this study, we have used the human glioma cell line U87 and a genetically modified variant of these cells (deficient in the signaling enzyme ERN1) to investigation the expression of different genes that encode retinoblastoma and retinoblastoma binding or associated proteins as well as the involvement of endoplasmic reticulum stress signaling system in the effect of hypoxia and glutamine or glucose deprivation on the expression of these gene. The level of suppression of the enzymatic activity of ERN1 was estimated by analysis of the expression of XBP1 and its splice variant (XBP1s) in U87 glioma cells that overexpress a dominant-negative construct of endoplasmic reticulum-nuclei-1 as compared to control glioma cells transfected with a vector. As shown in Figure 1, inductor of endoplasmic reticulum stress, tunicamycin $(10 \mu \mathrm{g} / \mathrm{ml})$, strongly induces the alternative splicing of XBP1 only in control glioma cells, while having no effect on this process in transfected by dnERN1 subline cells.

We have found that retinoblastoma and retinoblastoma like-1 genes as well as genes that encode retinoblastoma associated and binding proteins are expressed in the human glioma cell line U87 and the levels of expression of most of the studied genes are significantly depend from ERN1 signaling enzyme function. As shown in Figures 2 and $\mathbf{3}$, the expression levels of retinoblastoma and retinoblastoma like-1 mRNA in glioma cells, deficient in signaling enzyme ERN1, are increased by $31 \%$ and $96 \%$, respectively, as compared to control 1. Exposure of cells to hypoxia for $16 \mathrm{hrs}$ as well as to glutamine or glucose deprivation conditions leads to a decrease of retinoblastoma mRNA expression levels, but only in glioma cells with suppressed function of the signaling enzyme ERN1: $-21 \%,-46 \%$, and $-54 \%$, respectively, as compared to control 2 (Figure 2). However, no significant changes were found in control cells under these experimental 


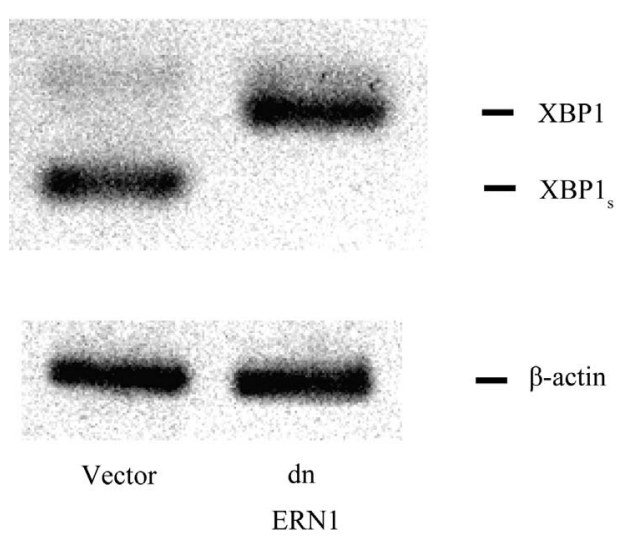

Figure 1. Effect of tunicamicin $(10 \mu \mathrm{g} / \mathrm{ml})$ on the expression of transcription factor XBP1 and its alternative splice variant (XBP1s) mRNA in glioma cell line U87 stable transfected with vector and its subline with blockade of signaling enzyme endoplasmic reticulum-nuclei-1 (ERN1) stable transfected with dnERN1.

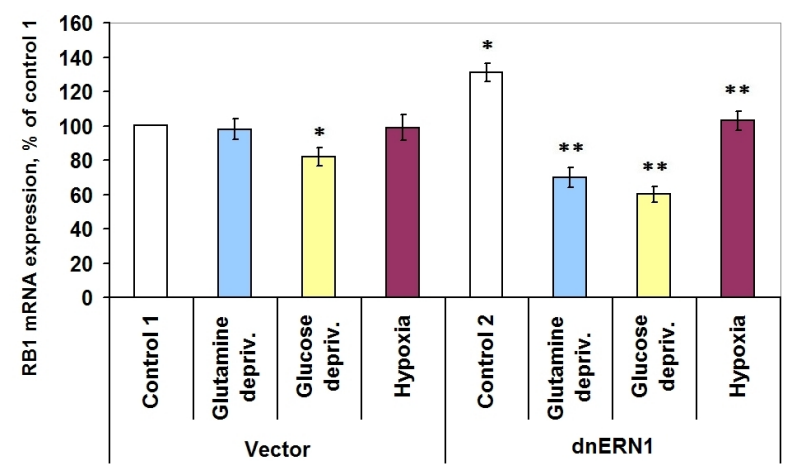

Figure 2. Effect of hypoxia and glucose or glutamine deprivation on the expression of retinoblastoma (RB1) mRNA in glioma cell line U87 (Vector) and its subline with a deficiency of the signaling enzyme ERN1 (dnERN1) measured by quantitative polymerase chain reaction. Values of RB1 mRNA expressions were normalized to the expression of beta-actin mRNA and represent as percent of control $(100 \%) ; n=3 ; * \mathrm{P}<0.05$ as compared to control $1 ; * * \mathrm{P}<0.05$ as compared to control 2 .

conditions.

At the same time, the level of retinoblastoma like-1 mRNA expression significantly decreased in both tested cell types under hypoxia, as well as under glutamine or glucose deprivation conditions: in control glioma cells: $-69 \%,-55 \%$, and $-50 \%$, respectively, as compared to control 1 and in the glioma cells subline with suppressed activity of signaling enzyme ERN1: $-56 \%,-70 \%$, and $-60 \%$, respectively, as compared to control 2 (Figure 3 ).

It was also shown that blockade of the signaling enzyme ERN1 does not change the expression level of mRNA of $95 \mathrm{kDa}$ retinoblastoma associated protein RNF40 (ring finger protein 40) which contains a RING finger, a motif known to be involved in protein-protein and protein-

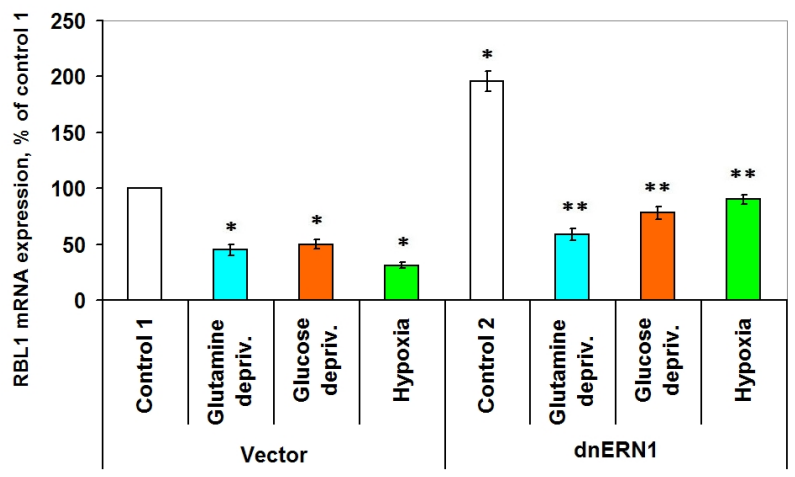

Figure 3. Effect of hypoxia and glucose or glutamine deprivation on the expression of retinoblastoma-like 1 (RBL1) mRNA in glioma cell line U87 (Vector) and its subline with a deficiency of the signaling enzyme ERN1 (dnERN1) measured by quantitative polymerase chain reaction. Values of RBL1 mRNA expressions were normalized to beta-actin mRNA expression and represent as percent for control $(100 \%) ; \boldsymbol{n}=3 ; * \mathrm{P}<0.05$ as compared to control $1 ; * * \mathrm{P}<0.05$ as compared to control 2 .

DNA interactions, and represents the E3 ubiquitin-protein ligase BRE1B (Figure 4). At the same time, we have also observed an increase $(+37 \%)$ of the expression level of $21 \mathrm{kDa}$ retinoblastoma-associated and apoptosis related protein EID-1 (EP300 interacting inhibitor of differentiation) mRNA in cells with knockdown of ERN1 as compared to control 1 (Figure 5). In glucose deprivation conditions no significant changes were found in the expression level of RNF40 mRNA in both glioma cell types, however, the expression level of this mRNA increases significantly in glutamine deprivation conditions in glioma cells with suppressed function of ERN1 enzyme (+51\%) (Figure 4). No significant changes were found in the expression level of EID1 mRNA in control glioma cells under hypoxia as well as in glutamine or glucose deprivation conditions, but significantly decreased in glioma cells with suppressed function of ERN1enzyme: $-12 \%$, $-33 \%$, and $-36 \%$, respectively (Figure 5).

As shown in Figures 6 and 7, the blockade of the function of the signaling enzyme ERN1 in glioma cells leads to a decrease of the expression level of mRNA of KDM5A (lysine-specific demethylase-5A) and a strong increase of histone demethylase JARID1B (lysine-specific demethylase-5B) mRNA, as compared to control 1. The expression levels of these mRNAs increase significantly in both tested cell types under hypoxia and in glutamine deprivation conditions. Thus, the expression levels of JARID1B mRNA increase in hypoxic and glutamine deprivation conditions in control glioma cells much more then in KDM5A: $+67 \%$ and $+96 \%$, respectively, for JARID1B and $+17 \%$ and $+18 \%$, respectively, for KDM5A as compared to control 1. Moreover, blockade of the function of the signaling enzyme ERN1 in glioma cells enhances the effect of hypoxia on the expression 


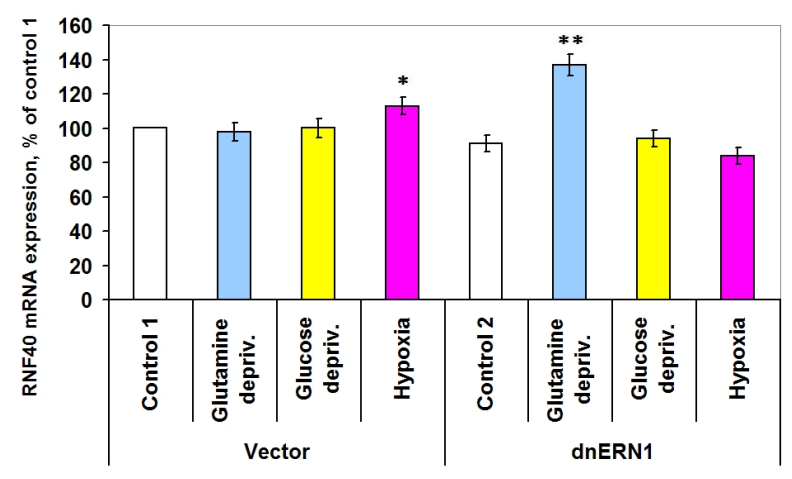

Figure 4. Effect of hypoxia and glucose or glutamine deprivation on the expression of retinoblastoma-associated protein RNF40 (ring finger protein 40) mRNA in glioma cell line U87 (Vector) and its subline with a deficiency of the signaling enzyme ERN1 (dnERN1) measured by quantitative polymerase chain reaction. Values of RNF40 mRNA expressions were normalized to beta-actin mRNA expression and represent as percent for control $(100 \%) ; n=3 ; * \mathrm{P}<0.05$ as compared to control $1 ; * * \mathrm{P}<0.05$ as compared to control 2 .

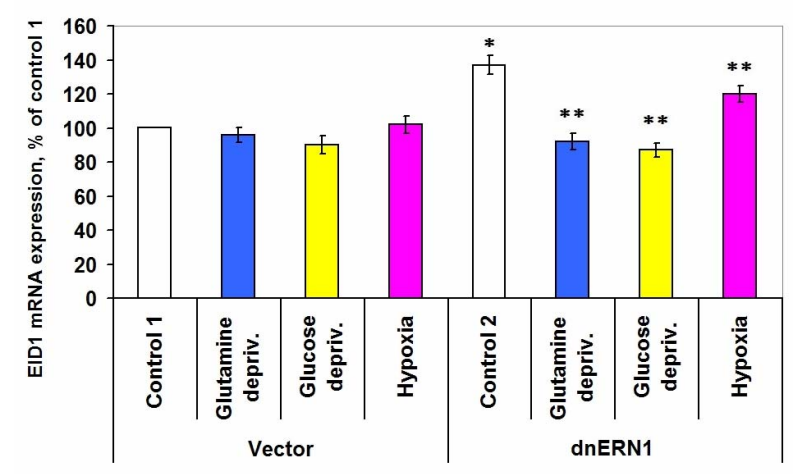

Figure 5. Effect of hypoxia and glucose or glutamine deprivation on the expression of retinoblastoma protein-associated protein EID1 (EP300 interacting inhibitor of differentiation 1) mRNA in glioma cell line U87 (Vector) and its subline with a deficiency of the signaling enzyme ERN1 (dnERN1) measured by quantitative polymerase chain reaction. Values of EID1 mRNA expressions were normalized to beta-actin mRNA expression and represent as percent for control $(100 \%) ; n=3$; $* \mathrm{P}<0.05$ as compared to control $1 ; * * \mathrm{P}<0.05$ as compared to control 2 .

levels of JARID1B mRNA (+126\% in ERN1 knockdown cells and $+18 \%$ in control glioma cells; Figure 7).

More significant changes were found in the expression levels of the transcription factor E2F1 mRNA, a retinoblastoma binding protein-3, in glioma cells with suppressed activity of signaling enzyme ERN1 (Figure 8). Thus, the expression level of E2F1 mRNA in these cells was more than 3 fold higher than in control glioma cells. Exposing cells to glutamine and glucose deprivation conditions leads to a decrease in the expression levels of E2F1 mRNA in both investigated cell types: $-60 \%$ and $-12 \%$, respectively, in control cells and $-62 \%$ and $-10 \%$,

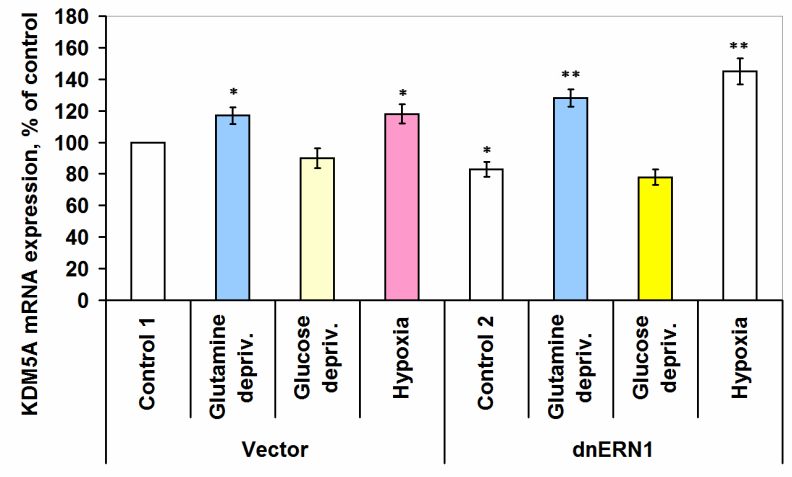

Figure 6. Effect of hypoxia and glucose or glutamine deprivation on the expression of KDM5A (lysine-specific demethylase $5 \mathrm{~A}$; retinoblastoma binding protein-2) mRNA in glioma cell line U87 (Vector) and its subline with a deficiency of the signaling enzyme ERN1 (dnERN1) measured by quantitative polymerase chain reaction. Values of KDM5A mRNA expressions were normalized to beta-actin mRNA expression and represent as percent for control $(100 \%) ; n=3 ; * \mathrm{P}<0.05$ as compared to control $1 ;{ }^{* *} \mathrm{P}<0.05$ as compared to control 2 .

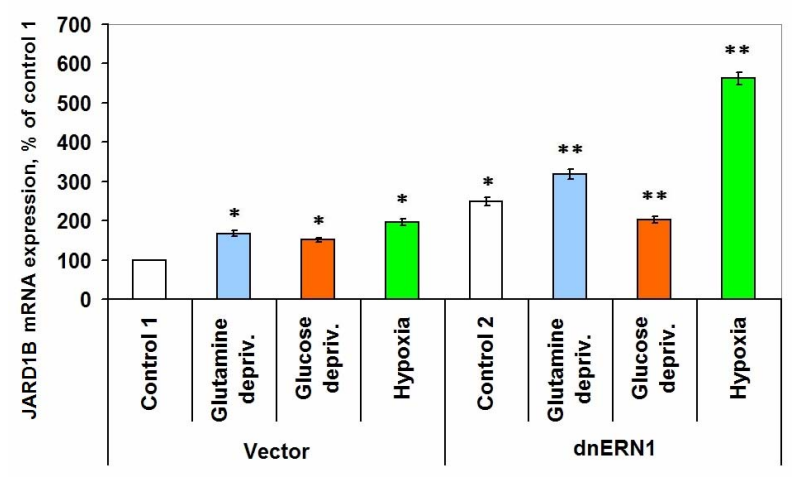

Figure 7. Effect of hypoxia and glucose or glutamine deprivation on the expression of JARD1B (lysine-specific demethylase $5 \mathrm{~B}$; retinoblastoma binding protein-2H1) mRNA in glioma cell line U87 (Vector) and its subline with a deficiency of the signaling enzyme ERN1 (dnERN1) measured by quantitative polymerase chain reaction. Values of JARD1B mRNA expressions were normalized to beta-actin mRNA expression and represent as percent for control $(100 \%) ; n=3 ; * \mathrm{P}<0.05$ as compared to control $1 ; * * \mathrm{P}<0.05$ as compared to control 2 .

respectively, in ERN1 knockdown cells. The results presented in Figure 8 have also shown that there is a 2 fold decreases in the expression levels of E2F1 mRNA under hypoxic conditions, but only in glioma cells with suppressed activity of the signaling enzyme ERN1 as compared to control 2.

As shown in Figure 9, the expression level of mRNA of transcription factor E2F3 significantly increased (2 fold) in glioma cells with suppressed activity of the signaling enzyme ERN1 as compared to control glioma cells (control 1). Exposing cells to glucose deprivation condition leads to a decrease in the expression levels of E2F3 


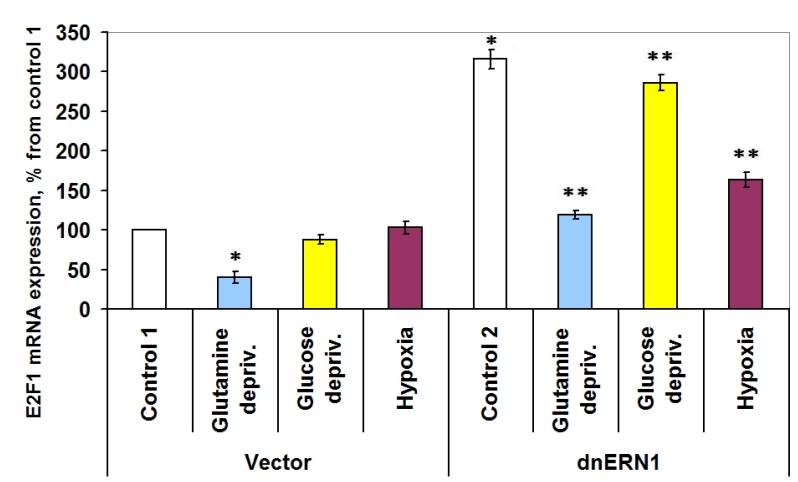

Figure 8. Effect of hypoxia and glucose or glutamine deprivation on the expression of transcription factor E2F1 mRNA in glioma cell line U87 (Vector) and its subline with a deficiency of the signaling enzyme ERN1 (dnERN1) measured by quantitative polymerase chain reaction. Values of E2F1 mRNA expressions were normalized to beta-actin mRNA expression and represent as percent for control $(100 \%) ; n=3 ; * \mathrm{P}<0.05$ as compared to control $1 ; * * \mathrm{P}<0.05$ as compared to control 2 .

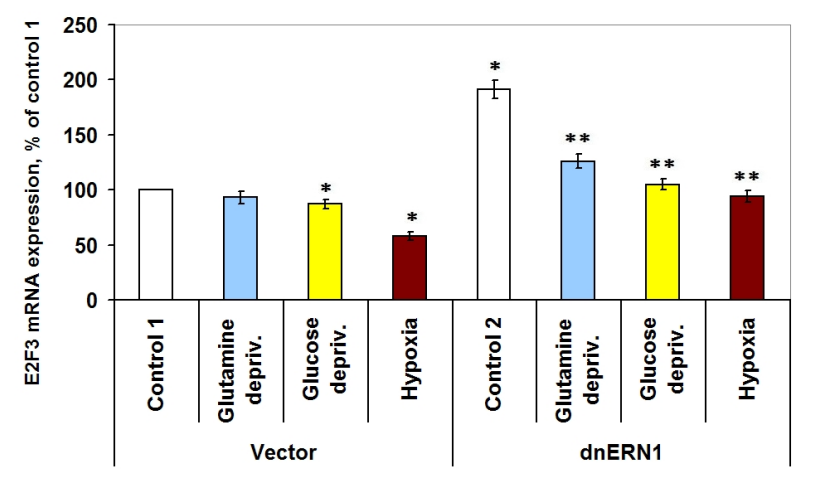

Figure 9. Effect of hypoxia and glucose or glutamine deprivation on the expression of transcription factor E2F3 mRNA in glioma cell line U87 (Vector) and its subline with a deficiency of the signaling enzyme ERN1 (dnERN1) measured by quantitative polymerase chain reaction. Values of E2F3 mRNA expressions were normalized to beta-actin mRNA expression and represent as percent for control $(100 \%) ; n=3 ; * \mathrm{P}<0.05$ as compared to control $1 ; * * \mathrm{P}<0.05$ as compared to control 2 .

mRNA in both investigated cell types: $-13 \%$ in control cells and $-45 \%$ in ERN1 knockdown cells. However, glutamine deprivation condition leads to decrease in the expression levels of this mRNA in glioma cells with suppressed activity of signaling enzyme ERN1 only. The results presented in Figure 9 also show that the expression levels of E2F3 mRNA decrease under hypoxic conditions both in control and ERN1 knockdown glioma cells, but inhibition of the ERN1 function enhances this effect.

Results of the investigation of expression levels of retinoblastoma binding proteins p48 (RBAP48) and p46 (RBAP46) mRNA are shown in Figures 10 and 11. Here, it was shown that the blockade of the activity of the sig- naling enzyme ERN1 leads to a significant induction of RBAP48 mRNA expression levels (+84\%) as compared to control glioma cells, which lack a significant change in the RBAP46 mRNA expression. We have, also, shown that the mRNA expression level of CTBP-interaction protein (CTIP) in glioma cells with suppressed activity of the signaling enzyme ERN1 significantly increases $(+50$ \%) as compared to control glioma cells (Figure 12). Exposure of glioma cells to medium without glutamine leads to decrease the expression levels of RBAP46 and

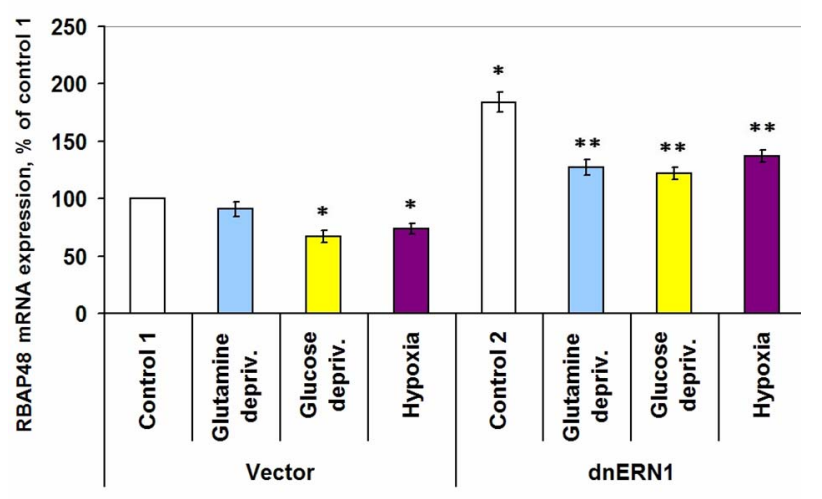

Figure 10. Effect of hypoxia and glucose or glutamine deprivation on the expression of RBAP48 (histone binding protein p48; chromatin assembly factor) mRNA in glioma cell line U87 (Vector) and its subline with a deficiency of the signaling enzyme endoplasmic reticulum-nuclei-1 (dnERN1) measured by quantitative polymerase chain reaction. Values of RBAP48 mRNA expressions were normalized to beta-actin mRNA expression and represent as percent for control $(100 \%) ; n=3 ; * \mathrm{P}$ $<0.05$ as compared to control $1 ; * * \mathrm{P}<0.05$ as compared to control 2.

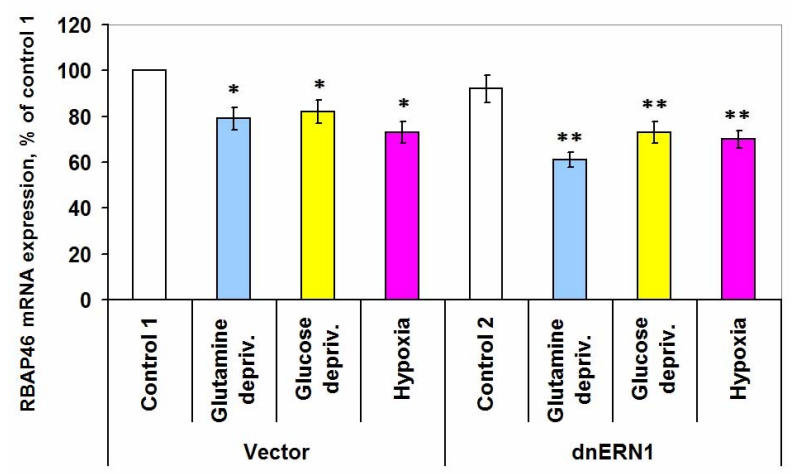

Figure 11. Effect of hypoxia and glucose or glutamine deprivation on the expression of RBAP46 (retinoblastoma binding protein $\mathrm{p} 46$; histone acetyltransferase type B) mRNA in glioma cell line U87 (Vector) and its subline with a deficiency of the signaling enzyme endoplasmic reticulum - nuclei-1 (dnERN1) measured by quantitative polymerase chain reaction. Values of RBAP46 mRNA expressions were normalized to beta-actin mRNA expression and represent as percent for control (100\%); $n=3 ; * \mathrm{P}<0.05$ as compared to control $1 ; * * \mathrm{P}<0.05$ as compared to control 2 . 
CTIP mRNA in control glioma cells $(-21 \%$ and $-22 \%$, respectively) as well as RBAP48, RBAP46, and CTIP mRNA in genetically modified cells: $-30 \%,-33 \%$, and $-33 \%$, respectively. However, during glucose deprivation conditions we have observed the decrease of the expression levels of mRNA of RBAP48 and RBAP46 only in both cell types: $-33 \%$ and $-18 \%$, respectively, in control cells and $-34 \%$ and $-21 \%$, respectively, in glioma cells with a blockade of the activity of ERN1 signaling enzyme (Figures 10 and 11). We have also shown that the expo- sure of glioma cells to hypoxic conditions leads to a significant decrease in the expression levels of RBAP48, RBAP46, and CTIP mRNA both in control glioma cells $(-26 \%,-27 \%$, and $-32 \%$, respectively) and in ERN1 knockdown cells $(-26 \%,-24 \%$, and $-46 \%$, respectively; Figures 10-12).

\section{DISCUSSION}

Bifunctional transmembrane signaling enzyme endoplasmic reticulum-nuclei-1 is a major proximal sensor of the unfolded protein response and participates in the early cellular response to the accumulation of misfolded proteins in the endoplasmic reticulum $[15,17]$. It has been known that the endoplasmic reticulum stress sensing, and signal transduction pathways are linked to the neovascularization process, tumor growth, and cellular death processes [17]. Moreover, the growing tumor requires endoplasmic reticulum stress, as well as ischemia and hypoxia both of which initiate endoplasmic reticulum stress, for own neovascularization and growth and for inhibition of apoptosis [15]. Therefore, in this work, we studied the expression of several genes that encode reti-noblastoma proteins and retinoblastoma binding or asso-

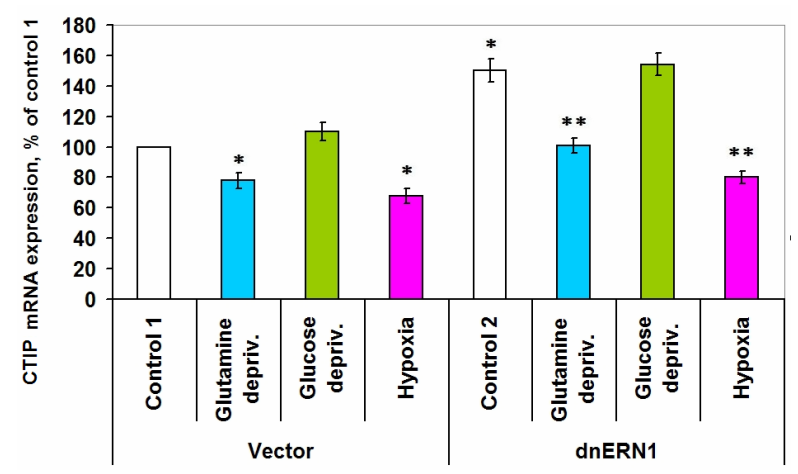

Figure 12. Effect of hypoxia and glucose or glutamine deprivation on the expression of CTIP (CTBP-interacting protein; DNA endonuclease) mRNA in glioma cell line U87 (Vector) and its subline with a deficiency of the signaling enzyme endoplasmic reticulum-nuclei-1 (dnERN1) measured by quantitative polymerase chain reaction. Values of CTIP mRNA expressions were normalized to beta-actin mRNA expression and represent as percent for control $(100 \%) ; n=3 ;{ }^{*} \mathrm{P}<0.05$ as compared to control $1 ; * * \mathrm{P}<0.05$ as compared to control 2 . ciated proteins in glioma cells with ERN1 knockdown for the purpose of evaluating the dependence of these genes upon the ERN1 signaling enzyme function. Results of this investigation clearly demonstrated that the expression levels of retinoblastoma proteins and most retinoblastoma binding or associated proteins, which form a complex with and function as a part of co-repressor complexes, increase in glioma cells without ERN1 signaling enzyme function. This data correlates with antitumor effects of this ERN1 blockade [15,17,35]. It is well known that retinoblastoma proteins, retinoblastoma binding and associated proteins as well as E2F transcription factors, cyclins, cyclin-dependent kinases and its inhibitors participate in the control of the cell cycle and proliferation and are the components of endoplasmic reticulum stress system $[14,19,22,36]$.

In this study we have studied the expression of genes that encode two retinoblastoma proteins and the family of retinoblastoma binding or associated proteins. These proteins are ubiquitously expressed nuclear proteins, among them E2F transcription factors, E3 ubiquitin-protein ligase BRE1B (RNF40), EP300 interacting inhibitor of differentiation EID1, lysine-specific histone demethylases KDM5A and JARD1B, histone acetyl transferase RBAP46 and DNA endonuclease CTIP, which participate in the control of cell cycle, proliferation, differentiation, and apoptosis and they play an important role in the control of malignant tumor growth $[14,15,23]$.

We have shown that blockade of the signaling activity of the enzyme endoplasmic reticulum-nuclei-1 leads to a significant induction of the expression of genes that encode tumor suppressor proteins retinoblastoma and transcription factors E2F1 and E2F3 which are possibly responsible for suppression of cell proliferation and tumor growth $[15,20,21]$. Thus, our data completely agrees with previous results where we showed that the complete blockade of endoplasmic reticulum-nuclei-1 signal transduction pathway had anti-tumor effects $[13,14]$. The antitumor effect of retinoblastoma proteins is mediated by the interaction with the cyclin D1 protein and the induction of its expression as well as by binding to the E2F transcription factors and growth suppression. Moreover, active complex E2F1 with differential protein-1 promotes apoptosis [22]. Thus, E2F1/DP1 induces the expression of ADP ribosylation factor, which in turn blocks ubiquination of p53. However, E2F1/DP1 complex can mediate p53-dependent apoptosis in the absence of ADP ribosylation factor through the upregulation of the ATM kinase and by enhancing p53 transcriptional activity.

We have also shown that the complete blockade of ERN1 signal transduction pathway leads to the induction of the expression of several genes which encode for retinoblastoma binding and associate proteins (EID1, JARID1B, RBAP48 and DNA endonuclease CTIP) that 
bind directly to retinoblastoma proteins to regulate cell proliferation, apoptosis, and tumor growth via retinoblastoma suppressor pathways [15,23,24]. These results also agree with anti-tumor effects of the complete blockade of ERN1 signaling pathway as well as with biological functions of the studied proteins $[13,14,25]$. The RBAP48 is present in protein complexes involved in histone acetylation and has been implicated in chromatin remodeling and transcriptional repression associated with histone deacetylation. It is important that this protein is also a part of the co-repressor complexes, which is an integral component of transcriptional silencing of oncogenes and suppression of carcinogenesis. We have also shown that DNA endonuclease CTIP mRNA expression is increased in ERN1 knockdown glioma cells and possibly contributes to suppression of glioma growth, because it is among several proteins that bind directly to the retinoblastoma protein, which regulates cell proliferation. CTIP is also associated with BRCA1 and is thought to modulate the functions of this protein: transcriptional regulation, DNA repair, and/or cell cycle checkpoint control [25].

In this study we have also shown that the exposure of cells to hypoxia and to a medium without glutamine or glucose leads to decrease in the expression level of mRNAs which encode for retinoblastoma like-1 protein and most of retinoblastoma binding proteins in control and in ERN1-deficient glioma cells. The expression levels of KDM5A (lysine-specific demethylase 5A) and JARD1B (lysine-specific demethylase 5B) increase mainly in control and ERN1 knockdown glioma cells exposed to hypoxia and glutamine deprivation condition. This phenomenon is probably due to induction of endoplasmic reticulum stress in hypoxic and nutrient starvation conditions and results in suppression of anti-proliferative proteins via endoplasmic reticulum stress signaling systems. It is possible that the expression levels of genes, which encode retinoblastoma proteins and most of retinoblastoma binding proteins, depends not only on ERN1 signaling enzyme, but also on two other signaling pathways: PERK (PKR-like endoplasmic reticulum kinase) and ATF6 (activating transcription factor 6), at least partly [4, 17].

The major finding reported here is that the expression of most tested genes that encode the retinoblastoma proteins and most of retinoblastoma binding proteins are dependent on the function of ERN1 signaling enzyme both in normal and hypoxic or nutrient deprivation conditions. It is possible that the retinoblastoma proteins and most of retinoblastoma binding or associated proteins participate in cell adaptive response to endoplasmic reticulum stress associated with ischemia or nutrient deprivation. However, the detailed molecular mechanisms of regulation of genes, which encode retinoblastoma proteins and retinoblastoma binding or associated proteins, by ERN1 signaling system under ischemic and nutrient deprivation conditions is complex and warrants further study.

\section{REFERENCES}

[1] Aragón, T., van Anken, E., Pincus, D., Serafimova, I.M., Korennykh, A.V., Rubio, C.A. and Walter, P. (2009) Messenger RNA targeting to endoplasmic reticulum stress signalling sites. Nature, 457, 736-740. doi:10.1038/nature07641

[2] Bi, M., Naczki, C., Koritzinsky, M., Fels, D., Blais, J., Hu, N., Harding, H., Novoa, I., Varia, M., Raleigh, J., Scheuner, D., Kaufman, R.J., Bell, J., Ron, D., Wouters, B.G. and Koumenis, C. (2005) ER stress-regulated translation increases tolerance to extreme hypoxia and promotes tumor growth. EMBO Journal, 24, 3470-3481. doi:10.1038/sj.emboj.7600777

[3] Blais, J.D., Filipenko, V., Bi, M., Harding, H.P., Ron, D., Koumenis, C., Wouters, B.G. and Bell, J.C. (2004) Transcription factor 4 is translationally regulated by hypoxic stress. Molecular and Cellular Biology, 24, 7469-7482. doi:10.1128/MCB.24.17.7469-7482.2004

[4] Fels, D.R. and Koumenis, C. (2006) The PERK/eIF2a/ ATF4 module of the UPR in hypoxia resistance and tumor growth. Cancer Biology and Therapy, 5, 723-728. doi:10.4161/cbt.5.7.2967

[5] Luo, D., He, Y., Zhang, H., Yu, L., Chen, H., Xu, Z., Tang, S., Urano, F. and Min, W. (2010) AIP1 is critical in transducing IRE1-mediated endoplasmic reticulum stress response. The Journal of Biological Chemistry, 283, 11905-11912. doi:10.1074/jbc.M710557200

[6] Korennykh, A.V., Egea, P.F., Korostelev, A.A., FinerMoore, J., Zhang, C., Shokat, K.M., Stroud, R.M. and Walter, P. (2009) The unfolded protein response signals through high-order assembly of Ire1. Nature, 457, 687693. doi: $10.1038 /$ nature 07661

[7] Romero-Ramirez, L., Cao, H., Nelson, D., Hammond, E., Lee, A.H., Yoshida, H., Mori, K., Glimcher, L.H., Denko, N.C., Giaccia, A.J., Le, Q.T. and Koong, A.C. (2004) XBP1 is essential for survival under hypoxic conditions and is required for tumor growth. Cancer Research, 64, 5943-5947. doi:10.1158/0008-5472.CAN-04-1606

[8] Lin, J.H., Li, H., Yasumura, D., Cohen, H.R., Zhang, C., Pannin, B., Shokat, K.M., Lavail, M.M. and Walter, P. (2007) IRE1 signaling affects cell fate during the unfolded protein response. Science, 318, 944-949. doi:10.1126/science.1146361

[9] Hollien, J., Lin, J.H., Li, H., Stevens, N., Walter, P. and Weissman, J.S. (2009) Regulated Ire1-dependent decay of messenger RNAs in mammalian cells. Journal of Cell Biology, 186, 323-331. doi:10.1083/jcb.200903014

[10] Acosta-Alvear, D., Zhou, Y., Blais, A., Tsikitis, M., Lents, N.H., Arias, C., Lennon, C.J., Kluger, Y. and Dynlacht, D.D. (2007) XBP1 controls diverse cell typeand condition-specific transcriptional regulatory networks. Molecular Cell, 27, 53-66. doi:10.1016/j.molcel.2007.06.011

[11] Han, D., Upton, J.-P., Hagen, A., Callahan, J., Oakes, S.A. 
and Papa, F.R. (2008) A kinase inhibitor activates the IRE1alpha RNase to confer cytoprotection against ER stress. Biochemical and Biophysical Research Communications, 365, 777-783. doi:10.1016/j.bbrc.2007.11.040

[12] Greenman, C., Stephans, P., Smith, R., Dalgliesh, G.L., Hunter, C., Bignell, G., Davies, H., et al. (2007) Patterns of somatic mutation in human genomes. Nature, 446, 153 -158. doi: $10.1038 /$ nature 05610

[13] Auf, G., Jabouille, A., Guérit, S., Pineau, R., Delugin, M., Bouchecareilh, M., Favereaux, A., Maitre, M., Gaiser, T., von Deimling, A., Czabanka, M., Vajkoczy, P., Chevet, E., Bikfalvi, A. and Moenner, M. (2010) A shift from an angiogenic to invasive phenotype induced in malignant glioma by inhibition of the unfolded protein response sensor IRE1. The Proceeding of the National Academy of Sciences of the United States of America, 107, 155515558.

[14] Drogat, B., Auguste, P., Nguyen, D.T., Bouchecareilh, M., Pineau, R., Nalbantoglu, J., Kaufman, R.J., Chevet, E., Bikfalvi, A. and Moenner, M. (2007) IRE1 signaling is essential for ischemia-induced vascular endothelial growth factor-A expression and contributes to angioge- nesis and tumor growth in vivo. Cancer Research, 67, 6700-6707. doi:10.1158/0008-5472.CAN-06-3235

[15] Moenner, M., Pluquet, O., Bouchecareilh, M. and Chevet, E. (2007) Integrated endoplasmic reticulum stress responses in cancer. Cancer Research, 67, 10631-10634. doi:10.1158/0008-5472.CAN-07-1705

[16] Denko, N.C. (2008) Hypoxia, HIF1 and glucose metabolism in the solid tumour. Nature Reviews Cancer, 8, 705-713. doi: $10.1038 / \mathrm{nrc} 2468$

[17] Saito, A., Ochiai, K., Kondo, S., Tsumagari, K., Murakami, T., Cavener, D.R. and Imaizumi, K. (2011) Endoplasmic Reticulum Stress Response Mediated by the PERK-eIF2-ATF4 Pathway Is Involved in Osteoblast Differentiation Induced by BMP2. The Journal of Biological Chemistry, 286, 4809-4818. doi:10.1074/jbc.M110.152900

[18] Hetz, C. and Glimcher, L.H. (2009) Fine-tuning of the unfolded protein response: Assembling the IRE1alpha interactome. Molecular Cell, 35, 551-561. doi:10.1016/j.molcel.2009.08.021

[19] Maddika, S., Ande, S.R., Panigrahi, S., Paranjothy, T., Weglarczyk, K., Zuse, A., Eshraghi, M., Manda, K.D., Wiechec, E. and Los, M. (2007) Cell survival, cell death and cell cycle pathways are interconnected: Implications for cancer therapy. Drug Resistance Updates, 10, 13-29. doi:10.1016/j.drup.2007.01.003

[20] Comstock, C.E., Augello, M.A., Schiewer, M.J., Karch, J., Burd, C.J., Ertel, A., Knudsen, E.S., Jessen, W.J., Aronow, B.J. and Knudsen, K.E. (2011) Cyclin D1 is a selective modifier of androgen-dependent signaling and androgen receptor function. The Journal of Biological Chemistry, 286, 8117-8127. doi:10.1074/jbc.M110.170720

[21] Liao, C.C., Tsai, C.Y., Chang, W.C., Lee, W.H. and Wang, J.M. (2010) RB.E2F1 complex mediates DNA damage responses through transcriptional regulation of ZBRK1. The Journal of Biological Chemistry, 285, 33134-33143. doi:10.1074/jbc.M110.143461
[22] Gurtner, A., Fuschi, P., Martelli, F., Manni, I., Artuso, S., Simonte, G., Ambrosino, V., Antonini, A., Folgiero, V., Falcioni, R., Sacchi, A. and Piaggio, G. (2010) Transcription factor NF-Y induces apoptosis in cells expressing wild-type p53 through E2F1 upregulation and p53 activation. Cancer Research, 70, 9711-9720. doi:10.1158/0008-5472.CAN-10-0721

[23] Sharma, A., Yeow, W.S., Ertel, A., Coleman, I., Clegg, N., Thangavel, C., Morrissey, C., Zhang, X., Comstock, C.E., Witkiewicz, A.K., Gomella, L., Knudsen, E.S., Nelson, P.S. and Knudsen, K.E. (2010) The retinoblastoma tumor suppressor controls androgen signaling and human prostate cancer progression. Journal of Clinical Investigation, 120, 4478-4492. doi:10.1172/JCI44239

[24] Kim, T.R., Lee, H.M., Lee, S.Y., Kim, E.J., Kim, K.C., Paik, S.G., Cho, E.W. and Kim, I.G. (2010) SM22alphainduced activation of $\mathrm{p} 16 \mathrm{INK} 4 \mathrm{a} /$ retinoblastoma pathway promotes cellular senescence caused by a subclinical dose of gamma-radiation and doxorubicin in HepG2 cells. Biochemical and Biophysical Research Communications, 400, 100-105. doi:10.1016/j.bbrc.2010.08.018

[25] Lopez-Bigas, N., Kisiel, T.A., Dewaal, D.C., Holmes, K.B., Volkert, T.L., Gupta, S., Love, J., Murray, H.L., Young, R.A. and Benevolenskaya, E.V. (2008) Genomewide analysis of the H3K4 histone demethylase RBP2 reveals a transcriptional program controlling differentiation. Molecular Cell, 31, 520-530.

doi:10.1016/j.molcel.2008.08.004

[26] Krishnakumar, R. and Kraus, W.L. (2010) PARP-1 regulates chromatin structure and transcription through a KDM5B-dependent pathway. Molecular Cell, 39, 736749. doi:10.1016/j.molcel.2010.08.014

[27] Hayami, S., Yoshimatsu, M., Veerakumarasivam, A., Unoki, M., Iwai, Y., Tsunoda, T., Field, H.I., Kelly, J.D., Neal, D.E., Yamaue, H., Ponder, B.A., Nakamura, Y. and Hamamoto R. (2010) Overexpression of the JmjC histone demethylase KDM5B in human carcinogenesis: Involvement in the proliferation of cancer cells through the E2F/RB pathway. Molecular Cancer, 9, 59 . doi:10.1186/1476-4598-9-59

[28] Li, R., Zhang, H., Yu, W., Chen, Y., Gui, B., Liang, J., Wang, Y., Sun, L., Yang, X., Zhang, Y., Shi, L., Li, Y. and Shang, Y. (2009) ZIP: A novel transcription repressor, represses EGFR oncogene and suppresses breast carcinogenesis. EMBO Journal, 28, 2763-2776. doi:10.1038/emboj.2009.211

[29] Creekmore, A.L., Walt, K.A., Schultz-Norton, J.R., Ziegler, Y.S., McLeod, I.X., Yates, J.R. and Nardulli A.M. (2008) The role of retinoblastoma-associated proteins 46 and 48 in estrogen receptor alpha mediated gene expression. Molecular and Cellular Endocrinology, 291, 79-86. doi:10.1016/j.mce.2008.05.016

[30] Salles, D., Mencalha, A.L., Ireno, I.C., Wiesmüller, L. and Abdelhay, E. (2011) BCR-ABL stimulates mutagenic homologous DNA double-strand break repair via the DNA-end-processing factor CtIP. Carcinogenesis, 32, 27-34. doi:10.1093/carcin/bgq216

[31] Kaidi, A., Weinert, B.T., Choudhary, C. and Jackson, S.P. (2010) Human SIRT6 promotes DNA end resection through CtIP deacetylation. Science, 329, 1348-1353. 
doi:10.1126/science.1192049

[32] Mueller, M.D., Vigne, J.-L., Minchenko, A.G., Lebovic, D.I., Leitman, D.C. and Taylor, R.N. (2000) Regulation of vascular endothelial growth factor (VEGF) gene transcription by estrogen receptors " $\alpha$ " and " $\beta$ ". The Proceeding of the National Academy of Sciences of the United States of America, 97, 10972-10977.

[33] Minchenko, A.G., Leshchinsky, I., Opentanova, I., Sang, N., Srinivas, V., Armstead, V.E. and Caro, J. (2002) Hypoxia-inducible factor-1-mediated expression of the 6-phosphofructo-2-kinase/fructose-2,6-bisphosphatase-3 (PFKFB3) gene. The Journal of Biological Chemistry, 277, 6183-6187. doi:10.1074/jbc.M110978200

[34] Kulinich, A.O., Minchenko, D.O., Maslak, A.S., Shevtsova, A.I., Brazaluk, O.Z. and Minchenko, O.H. (2010)
Fibronectin-1 expression in lymphocytes of patients with erythremia disease. The Ukrainian Bioche- mical Journal, 82, 53-59.

[35] Petibois, C., Drogat, B., Bikfalvi, A., Deleris, G. and Moenner, M. (2007) Histological mapping of biochemical changes in solid tumors by FT-IR spectral imaging. FEBS Letters, 581, 5469-5474.

[36] Minchenko, D.M., Hubenya, O.V., Terletsky, B.M., Moenner, M. and Minchenko, O.H. (2011) Effect of hypoxia, glutamine and glucose deprivation on the expression of cyclin and cyclin-dependent kinase genes in glioma cell line U87 and its subline with suppressed activity of signaling enzyme endoplasmic reticulumnuclei-1. The Ukrainian Biochemical Journal, 83, 5-16. 